\title{
EDITORIAL
}

\section{MicroRNA immunobiology: when microRNA chemists meet immunologists}

Cellular \& Molecular Immunology (2011) 8, 369-370; doi:10.1038/cmi.2011.32; published online 22 August 2011

A lthough interdisciplinary research has been heralded as the engine of basic discovery for decades, many in the immunology community have been taken back by the recent marriage between microRNA (miRNA) and immunology. MicroRNAs were first discovered by Ambros and colleagues in 1993. ${ }^{1}$ They are small untranslated RNAs, highly conserved between different eukaryotic species. ${ }^{2-5}$ They are encoded by specific genes in the genome, which are controlled at the transcriptional level in a manner similar to protein-encoding genes. ${ }^{2}$ Following the synthesis of the primary miRNA by RNA polymerase II or III, nuclear processing by the enzyme Drosha produces a primary miRNA transcript which can be shuttled into the cytoplasm ${ }^{2}$ Final production of the mature miRNA species requires further cytoplasmic processing by an RNase III enzyme called Dicer, producing a 19- to 24-base pair product, capable of being incorporated into the RNA-induced silencing complex. The RNA-induced silencing complex, in turn, is able to use the 'seed sequence' of the miRNA to recognize complementary mRNA transcripts for degradation or translational silencing. To date, more than 800 human miRNAs have been identified, regulating an estimated $50 \%$ of all human genes. Each miRNA appears to regulate the expression of tens to hundreds of genes, thereby functioning as 'master-switches' that regulate and coordinate multiple cellular pathways in important processes such as embryonic development and oncogenesis, as well as cellular growth and proliferation. ${ }^{2-5}$

Inspired by these discoveries, a number of laboratories have recently investigated the potential roles of miRNAs in immune cells. This has led to the unexpected finding that miRNAs are crucial for essentially all major immune processes. For example, Toll-like receptors (TLRs) induce the expression of more than 20 miRNAs (including miR-21, miR-15b, miR-16, miR-23b, miR-27b, miR-30b, miR-30c, miR-125b, miR-146, miR-147 and miR-155), each of which selectively targets a specific group of mRNAs. ${ }^{6-10}$ Thus, TLR-induced miR-146a turns off TLR response by degrading IRAK1 and TRAF6 mRNAs that are required for TLR signaling, and mediates lipopolysaccharide tolerance that is crucial for preventing septic shock ${ }^{6,11}$ Similarly, TLR4-induced miR-21 blocks TLR4 response by targeting programmed cell death 4 , a crucial transcriptional and translational regulator of TLR-induced gene expression ${ }^{12}$ Knocking down either miR-146a or miR-21, or knocking out dicer,

Correspondence: Dr YH Chen, 713 Stellar-Chance Laboratories, Department of Pathology and Laboratory Medicine, University of Pennsylvania School of Medicine, 422 Curie Blvd., Philadelphia, PA 19104, USA.

E-mail: yhc@mail.med.upenn.edu

Received 19 July 2011; accepted 20 July 2011 a gene that controls miRNA biogenesis, leads to hyper-activation of TLRs $^{6,9,12}$ Thus, besides the negative regulatory proteins of TLRs, ${ }^{13}$ TLR-induced microRNAs represent a new class of negative feedback regulators essential for preventing deleterious inflammatory conditions induced by pathogens.

In this special issue of Cellular and Molecular Immunology, we provide readers with three expert reviews surveying the most recent advances in this nascent field. Specifically, Mi and colleagues will discuss the roles of miRNAs in the development and function of $\mathrm{CD} 4{ }^{+} \mathrm{CD} 25^{+} \mathrm{Foxp}^{+}$regulatory $\mathrm{T}$ cells and invariant natural killer $\mathrm{T}$ cells, two cell subsets that are critical for maintaining immune homeostasis and tolerance. Chan and colleagues will focus on the roles of microRNAs in TLR signaling and endotoxin tolerance; the functions of TLR ligand-induced microRNAs, including miR-146a, miR155 and miR-132, in regulating immunity and tolerance will be discussed in details. Finally, Chen and colleagues bring us to the mucosal surface, where epithelial cells meet with miRNAs as well as pathogens; miRNAs may modulate epithelial immune responses at multiple levels, including production and release of cytokines/chemokines, expression of adhesion and costimulatory molecules, and feedback regulation of immune homeostasis.

However, because miRNA immunobiology is a nascent field, many fundamental questions related to miRNA in immunity have yet to be answered. It is unknown how immune receptors selectively activate some miRNA genes but not others; once activated, it is unclear how miRNAs selectively control immune responses that induce them. TLRs can upregulate $>20$ miRNAs and downregulate $>30^{6-10}$ As many of these miRNAs regulated by TLR signaling are also dysregulated in cancer, it is possible that miRNAs form a key link between inflammation and cancer and that the induction of specific miRNAs, including miR-21, by TLRs may be a key step in tumor genesis. Thus, interdisciplinary research will likely continue to be the engine of growth of this nascent field for years to come.

Youhai H Chen

Department of Pathology and Laboratory of Medicine, University of Pennsylvania School of Medicine, Philadelphia,

$P A, U S A$

1 Lee RC, Feinbaum RL, Ambros V. The C. elegans heterochronic gene lin-4 encodes small RNAs with antisense complementarity to lin-14. Cell 1993; 75: 843-854. 
2 Carthew RW, Sontheimer EJ. Origins and mechanisms of miRNAs and siRNAs. Cell 2009; 136: 642-655.

3 Nelson P, Kiriakidou M, Sharma A, Maniataki E, Mourelatos Z. The microRNA world: small is mighty. Trends Biochem Sci 2003; 28: 534-540.

4 Shomron N, Levy C. MicroRNA-biogenesis and pre-mRNA splicing crosstalk. J Biomed Biotechnol 2009; 2009594678.

5 Chua JH, Armugam A, Jeyaseelan K. MicroRNAs: biogenesis, function and applications. Curr Opin Mol Ther 2009; 11: 189-199.

6 Taganov KD, Boldin MP, Chang KJ, Baltimore D. NF-kappaB-dependent induction of microRNA miR-146, an inhibitor targeted to signaling proteins of innate immune responses. Proc Natl Acad Sci USA 2006; 103: 12481-12486.

7 Liu G, Friggeri A, Yang Y, Park YJ, Tsuruta Y, Abraham E. MiR-147, a microRNA that is induced upon Toll-like receptor stimulation, regulates murine macrophage inflammatory responses. Proc Natl Acad Sci USA 2009; 106: 15819-15824.

8 Ceppi M, Pereira PM, Dunand-Sauthier I, Barras E, Reith W, Santos MA et al. MicroRNA-155 modulates the interleukin-1 signaling pathway in activated human monocyte-derived dendritic cells. Proc Natl Acad Sci USA 2009; 106: 2735-2740.

9 Ruggiero T, Trabucchi M, de Santa F, Zupo S, Harfe BD, McManus MT et al. LPS induces $\mathrm{KH}$-type splicing regulatory protein-dependent processing of microRNA-155 precursors in macrophages. FASEB J 2009; 23: 2898-2908.

10 Zhou R, Hu G, Liu J, Gong A, Drescher KM, Chen XM. NF-kappaB p65-dependent transactivation of miRNA genes following Cryptosporidium parvum infection stimulates epithelial cell immune responses. PLoS Pathog 2009; 5 e1000681.

11 Nahid MA, Pauley KM, Satoh M, Chan EK. MiR-146a is critical for endotoxininduced tolerance: implication in innate immunity. J Biol Chem 2009; 284: 34590-34599.

12 Sheedy FJ, Palsson-McDermott E, Hennessy EJ, Martin C, O'Leary JJ, Ruan Q et al. Negative regulation of TLR4 via targeting of the proinflammatory tumor suppressor PDCD4 by the microRNA miR-21. Nat Immunol 2010; 11: 141-147.

13 Liew FY, Xu D, Brint EK, O'Neill LA. Negative regulation of Toll-like receptor-mediated immune responses. Nat Rev Immunol 2005; 5: 446-458. 